\title{
An Approach to Distributed Min-Max Model Predictive Control of Linear Systems with Parametric Uncertainty ${ }^{1}$
}

Key Words: Min-max model predictive control; distributed control; linear interconnected systems; polytopic uncertainty.

Abstract. In this paper, a suboptimal approach to distributed closed-loop min-max MPC for uncertain systems consisting of polytopic subsystems with coupled dynamics subject to both state and input constraints is proposed. The approach applies the dynamic dual decomposition method and reformulates the original centralized min-max MPC problem into a distributed optimization problem. The suggested approach is illustrated on a simulation example of an uncertain system consisting of two interconnected polytopic subsystems.

\section{Introduction}

Model Predictive Control (MPC) is an efficient methodology to solve complex constrained multivariable control problems in the absence, as well as in the presence
A. Grancharova, S. Olaru

subject to the system dynamics, and state and input constraints. The conceptual structure of MPC is given in figure 1 [1]. The MPC denomination stems from the idea of employing an explicit model of the plant to be controlled which is used to predict the future output behaviour. This prediction capability allows solving optimal control problems on line, where tracking error, namely the difference between the predicted output and the desired reference, is minimized over a future horizon [1].

The result of the optimization is applied according to a receding or moving horizon philosophy [1]: At time $t$ only the first input of the optimal command sequence is actually applied to the plant. The remaining optimal inputs are discarded, and a new optimal control problem is solved at time $t+1$.

The model-based philosophy is the major advantage of the MPC philosophy but brings also the most important difficulties. Since the model is only an approximation of the

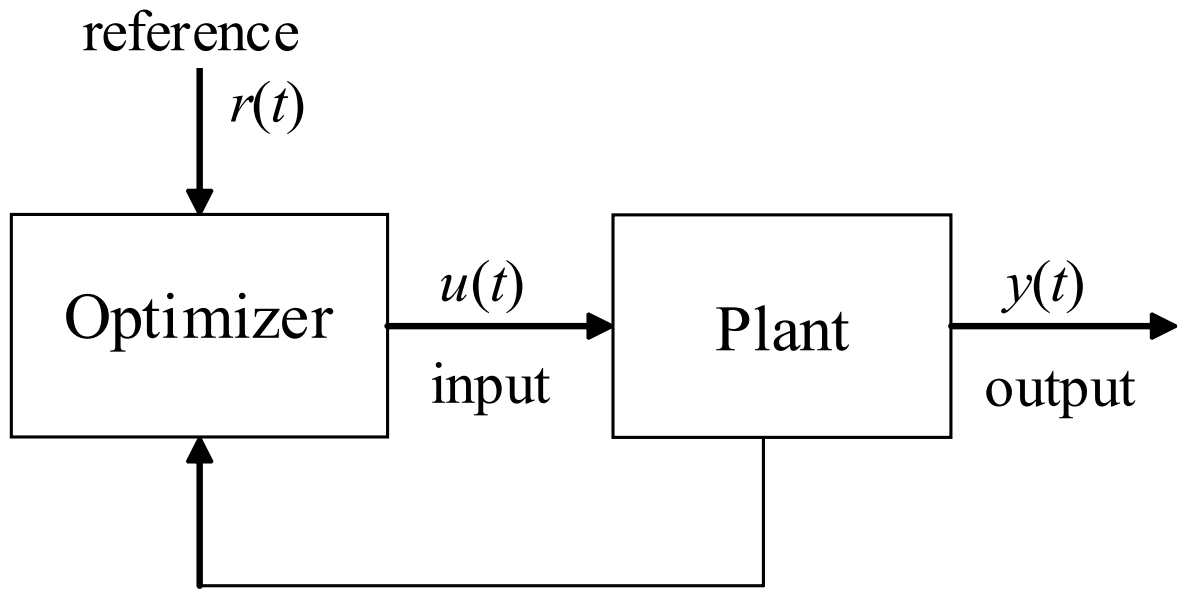

measurements

Figure 1. Basic MPC structure [1]

of uncertainties [1-4]. MPC involves the solution at each sampling instant of a finite horizon optimal control problem

\footnotetext{
${ }^{1}$ This work was financed by the National Science Fund of the Ministry of Education and Science of Republic of Bulgaria, contract No. DRila 01/12 and the Partenariats Hubert Curien (PHC) Programme Rila of the French Government, contract No. 29401 YJ "Robust distributed predictive control of complex systems", as well as by the Scientific-Research Sector of the University of Chemical Technology and Metallurgy, Sofia, Bulgaria.
}

real plant, it is important for the MPC to be robust with respect to model uncertainties and plant disturbances [1]. One possible strategy to obtain this is the design of minmax MPC, as was first proposed in [5]. There are two formulations of min-max MPC: the open-loop formulation (the optimization is performed over a sequence of control actions) and the closed-loop formulation (the optimization is performed over a sequence of feedback control laws) (see [6] for a review of the min-max MPC approaches). The open-loop min-max MPC [6] guarantees the robust stability 


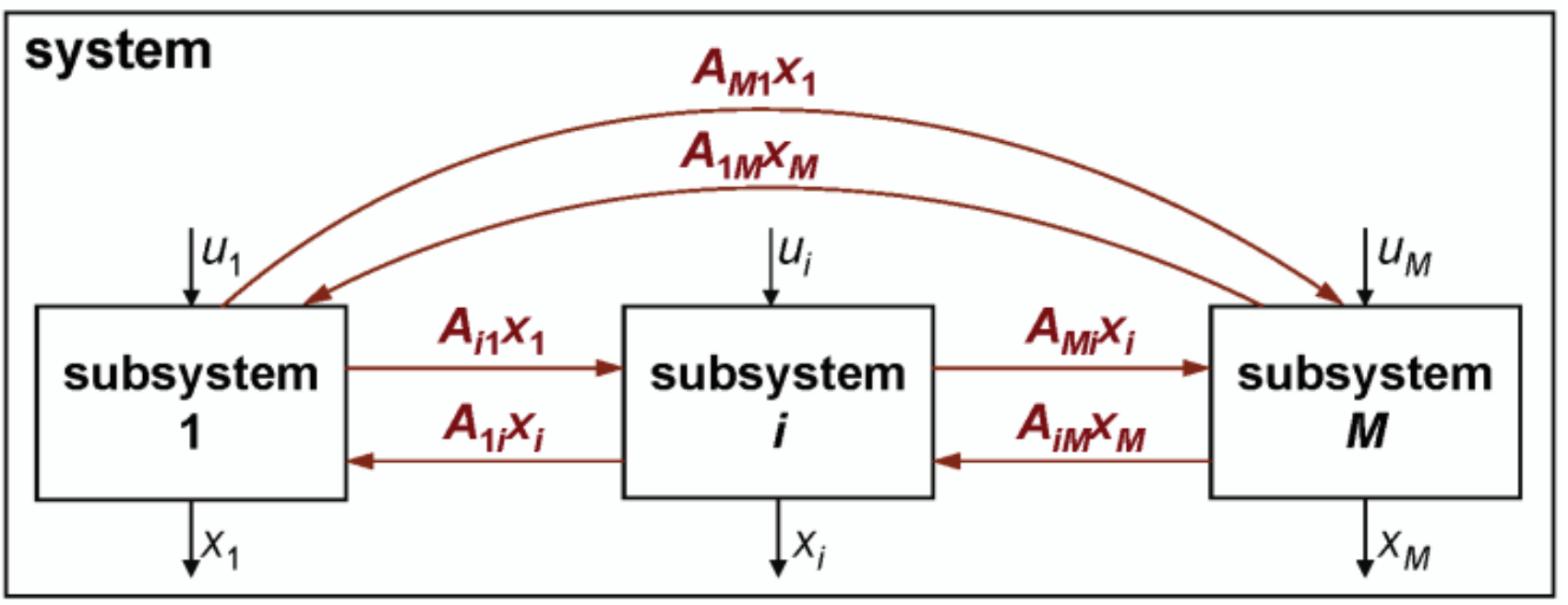

Figure 2. System composed by the interconnection of $M$ subsystems

and the robust feasibility of the system, but it may be very conservative since the control sequence has to ensure constraints fulfilment for all possible uncertainty scenarios without considering the fact that future measurements of the state contain information about past uncertainty values. The conservativeness of the open-loop approaches can be overcome by the closed-loop min-max MPC approaches [6].

However, solving in a centralized way MPC problems for medium- and large-scale systems may be impractical due to the large number of decision variables and the topology of the plant and data communication. Therefore, there is a strong motivation for development of methods for distributed solution of MPC problems. The distributed MPC has the advantage to reduce the original, large size, optimization problem into a number of smaller and more tractable ones. Recently, several approaches for distributed /decentralized MPC have been proposed $[7,8,9]$.

There are only a few papers considering the problem of robust distributed MPC of interconnected polytopic systems. Thus, in [10] a distributed MPC algorithm for polytopic systems subject to actuator saturation is proposed. In [11], an online distributed MPC algorithm that deals explicitly with model errors is proposed. It is supposed that the subsystems are coupled through their inputs and only constraints on the inputs are considered. In [12,13], an approach to distributed open-loop min-max MPC for interconnected polytopic systems is developed, which is based on the dual decomposition method [14,15].

In this paper, an approach to distributed closed-loop min-max MPC for uncertain linear systems consisting of polytopic subsystems with coupled dynamics subject to both state and input constraints is proposed. The approach applies the dynamic dual decomposition method [14,15] and reformulates the centralized closed-loop min-max MPC problem into a distributed closed-loop min-max MPC problem. It is based on distributed on-line optimization and can be applied to large-scale polytopic systems. In comparison with $[12,13]$, here the optimization of the feedback policies is described as an alternative to the optimization of the control actions.

\section{Formulation of the Closed-Loop Min-Max MPC Problem}

Consider a system composed by the interconnection of $M$ linear uncertain subsystems (figure 2), described by the polytopic discrete-time models:

(1)

$$
\begin{aligned}
& x_{i}(t+1)=A_{i}(t) x_{i}(t)+B_{i}(t) u_{i}(t)+\sum_{\substack{j=1 \\
j \neq i}}^{M} A_{i j} x_{j}(t) \\
& {\left[A_{i}(t), B_{i}(t)\right] \in \Omega_{i}} \\
& i=1,2, \ldots, M
\end{aligned}
$$

where $x_{i}(t) \in \mathfrak{R}^{n_{i}}$ and $u_{i}(t) \in \mathfrak{R}^{m_{i}}$ are the state and control input vectors, related to the $i$-th subsystem, $A_{i}(t) \in \mathfrak{R}^{n_{i} \times n_{i}}$ and $B_{i}(t) \in \mathfrak{R}^{n_{i} \times m_{i}}$ are uncertain time-varying matrices, and $A_{i j} \in \Re^{n_{i} \times n_{i}} j=1,2, \ldots, M, j \neq i$ are known constant martices.

For a polytopic uncertainty description, $\Omega_{i}$ $i=1,2, \ldots, M$ are polytopes

(2) $\Omega_{i}=\operatorname{Co}\left\{\left[A_{i}^{1}, B_{i}^{1}\right],\left[A_{i}^{2}, B_{i}^{2}\right], \ldots,\left[A_{i}^{L_{i}}, B_{i}^{L_{i}}\right]\right\}, i=1, \ldots, M$

where $C o\{\cdot\}$ denotes convex hull and $\left[A_{i}^{r}, B_{i}^{r}\right], r=1,2, \ldots, L_{i}$ are its vertices. The following constraints are imposed on the subsystems:

(3) $u_{\min , i} \leq u_{i}(t) \leq u_{\max , i}, x_{\min , i} \leq x_{i}(t) \leq x_{\max , i}, i=1, \ldots, M$

The following assumption is made:

A1. $x_{\min , i}<0<x_{\max , i}, u_{\min , i}<0<u_{\max , i}, i=1, \ldots, M$.

Let $x(t)$ and $u(t)$ denote the overall state and the overall control input, i.e.:

(4)

$$
\begin{aligned}
& x(t)=\left[x_{1}(t), x_{2}(t), \ldots, x_{M}(t)\right] \in \Re^{n}, n=\sum_{i=1}^{M} n_{i} \\
& u(t)=\left[u_{1}(t), u_{2}(t), \ldots, u_{M}(t)\right] \in \Re^{m}, m=\sum_{i=1}^{M} m_{i}
\end{aligned}
$$

Another assumption will be made with respect to the rate of variation of parameters, mainly with respect to the 
prediction horizon as it will be shown next in the MPC design.

A2. The uncertain pairs $\left[A_{i}(t), B_{i}(t)\right] \in \Omega_{i}, i=1, \ldots, M$ have infrequent changes in the sense that $\left[A_{i}(t), B_{i}(t)\right]=$ const, $i=1, \ldots, M$ for periods of time, which are not less than $\tilde{N}$ ( $\tilde{N} \in \mathbb{N}$ is supposed to be sufficiently large).

Before formulating the robust MPC problem, a set $\tilde{\Omega}_{i}$ is introduced, which is a finite subset of $\Omega_{i}$. The set $\tilde{\Omega}_{i}$ is defined by:

(6) $\tilde{\Omega}_{i}=\Omega_{i}^{\mathrm{vert}} \cup \Omega_{i}^{\mathrm{int}}$

where $\Omega_{i}^{\text {vert }}=\left\{\left[A_{i}^{r}, B_{i}^{r}\right], r=1,2, \ldots, L_{i}\right\}$ is the set of vertices of $\Omega_{i}$ and $\Omega_{i}^{\text {int }}=\left\{\left[A_{i}^{L_{i}+j}, B_{i}^{L_{i}+j}\right] \in \operatorname{int}\left(\Omega_{i}\right), j=1,2, \ldots, K_{i}\right\}$ is a finite set which includes interior points of the set $\Omega$.

It is supposed that a full measurement $x=\left[x_{1}, x_{2}, \ldots, x_{M}\right]$ of the overall state is available at the current time $t$. The robust regulation problem is considered where the goal is to steer the overall state of the system (1) to the origin. Let $N$ be a finite horizon such that $N<\tilde{N}$. The Assumption A2 characterizes a framework under which the model has a slow variation with respect to the time constants and prediction horizons. Thus, it can be accepted that $\left[A_{i}(t+k), B_{i}(t+k)\right]=$ $=$ const $=\left[A_{i}, B_{i}\right], k=0,1, \ldots, N, i=1, \ldots, M$. Let $F_{i}=\left[f_{i, 0}, f_{i, 1}, \ldots, f_{i, N-1}\right]$ be a sequence of feedback laws for the $i$-th subsystem, where $f_{i, k}: \mathfrak{R}^{n_{i}} \rightarrow \mathfrak{R}^{m_{i}}, k=0,1, \ldots, N-1$ are known functions. Let $F=\left[F_{1}, F_{2}, \ldots, F_{M}\right]$, $F: \mathfrak{R}^{N n} \rightarrow \mathfrak{R}^{N m}$ contains the feedback laws for the whole system. Then, for the current $x$, the robust regulation MPC solves the optimization problem:

\section{Problem P1 (centralized min-max MPC)}

$$
V^{*}(x)=\min _{F} \max _{\substack{\left[A_{i}, B_{i}\right] \in \tilde{\Omega}_{i} \\ i=1,2, \ldots, M}} J\left(U, x,\left[A_{1}, B_{1}\right], \ldots,\left[A_{M}, B_{M}\right]\right)
$$

subject to $x_{t \mid t}=x$ and

(8) $x_{i, t+k \mid t} \in \mathrm{X}_{i}, \forall\left[A_{i}, B_{i}\right] \in \tilde{\Omega}_{i}, i=1, \ldots, M, k=1, \ldots, N$

(9) $u_{i, t+k} \in U_{i}, i=1, \ldots, M, k=0,1, \ldots, N-1$

(10) $u_{i, t+k}=f_{i, k}\left(x_{i, t+k \mid t}\right), k=0,1, \ldots, N-1$

$$
\begin{aligned}
& x_{i, t+k+1 \mid t}=A_{i} x_{i, t+k \mid t}+B_{i} u_{i, t+k}+\sum_{j=1, j \neq i}^{M} A_{i j} x_{j, t+k \mid t} \\
& {\left[A_{i}, B_{i}\right] \in \tilde{\Omega}_{i}, i=1, \ldots, M, k=0,1, \ldots, N-1}
\end{aligned}
$$

(12) $x_{t+k \mid t}=\left[x_{1, t+k \mid t}, x_{2, t+k \mid t}, \ldots, x_{M, t+k \mid t}\right], k=0,1, \ldots, N$

(13) $f_{t+k}=\left[f_{1, k}, f_{2, k}, \ldots, f_{M, k}\right], k=0,1, \ldots, N-1$

where $F=\left[f_{t}, f_{t+1}, \ldots, f_{t+N}\right]$. The cost function is

(14) $J\left(U, x,\left[A_{1}, B_{1}\right], \ldots,\left[A_{M}, B_{M}\right]\right)=\sum_{k=0}^{N} l\left(x_{t+k \mid t}, u_{t+k}\right)$

where

$$
l\left(x_{t+k \mid t}, u_{t+k}\right)=\sum_{i=1}^{M} l_{i}\left(x_{i, t+k \mid t}, u_{i, t+k}\right)
$$

Here, $l_{i}\left(x_{i, t+k \mid t}, u_{i, t+k}\right)=x_{i, t+k \mid t}^{T} Q_{i} x_{i, t+k \mid t}+u_{i, t+k}^{T} R_{i} u_{i, t+k \mid t}$ is the stage cost for the $i$-th subsystem and $Q_{i}, R_{i} \succ 0$ are weighting matrices. The sets $\mathrm{X}_{i}$ and $\mathrm{U}_{i}$ are defined by

(16) $\mathrm{X}_{i}=\left\{x_{i} \in \mathfrak{R}^{n_{i}} \mid x_{\min , i} \leq x_{i} \leq x_{\max , i}\right\}$

(17) $\mathrm{U}_{i}=\left\{u_{i} \in \mathfrak{R}^{m_{i}} \mid u_{\min , i} \leq u_{i} \leq u_{\max , i}\right\}$

It follows from (16)-(17) that $\mathrm{X}_{i}$ and $\mathrm{U}_{i}$ are convex (polyhedral) sets, which include the origin in their interior (due to Assumption A1). It should be noted that the state constraints (8) guarantee the robust feasibility of the solution in sense that the state constraints in (3) will be satisfied for the worst-case uncertainty realizations in $\tilde{\Omega}_{i}$, $i=1, \ldots, M$.

Let $F^{*}=\left[f_{0}^{*}, f_{1}^{*}, \ldots, f_{N-1}^{*}\right]$ denote the optimal solution of problem P1. According to the receding horizon strategy, the control action applied to the plant at time $t$ is $u\left(x_{t \mid t}\right)=f_{0}^{*}\left(x_{t \mid t}\right)$. The following assumption is also made:

A3. Each control law $f_{i, k}\left(x_{i}\right), i=1, \ldots, M$, $k=0,1, \ldots, N-1$ is linear, i.e. it has the form:

(18) $f_{i, k}\left(x_{i, t+k \mid t}\right)=G_{i, t+k} x_{i, t+k \mid t}+g_{i, t+k}$

where the matrix $G_{i, t+k} \in \mathfrak{R}^{m_{i} \times n_{i}}$ and the vector $g_{i, t+k} \in \mathfrak{R}^{m_{i}}$ are to be optimized.

Let $G=\left[G_{1}, G_{2}, \ldots, G_{M}\right]$ and $g=\left[g_{1}, g_{2}, \ldots, g_{M}\right]$, where

$$
\begin{aligned}
& G_{i}=\left[G_{i, t}, G_{i, t+1}, \ldots, G_{i, t+N}\right] \\
& g_{i}=\left[g_{i, t}, g_{i, t+1}, \ldots, g_{i, t+N}\right] \\
& i=1,2, \ldots, M
\end{aligned}
$$

By taking into account (18)-(19), the equation (7) obtains the form

$$
V^{*}(x)=\min _{G, g} \max _{\substack{\left[A_{i}, B_{i}\right] \in \tilde{\Omega}_{i} \\ i=1,2, \ldots, M}} J\left(U, x,\left[A_{1}, B_{1}\right], \ldots,\left[A_{M}, B_{M}\right]\right)
$$

It should be noted that the cost function (14) is in general non-convex with respect to the uncertain matrices $A_{i}, B_{i}, i=1, \ldots, M$. Therefore, considering only the vertices of the sets $\Omega_{i}, i=1, \ldots, M$ when computing the worst-case cost would not have been sufficient. For this reason, the finite uncertainty sets $\tilde{\Omega}_{i}$, defined by (6) include some interior elements in addition to the vertices.

\section{Distributed Closed-Loop Min-Max MPC of Linear Systems with Uncertainty}

Problem P1 can be decomposed by using the dynamic dual decomposition approach $[4,5]$. The following decoupled state equations can be formulated:

$$
\begin{aligned}
& x_{i}(t+1)=A_{i}(t) x_{i}(t)+B_{i}(t) u_{i}(t)+v_{i}(t) \\
& {\left[A_{i}(t), B_{i}(t)\right] \in \Omega_{i}} \\
& i=1,2, \ldots, M
\end{aligned}
$$

with the additional constraints that [5] 
(22)

$$
v_{i}(t)=\sum_{\substack{j=1 \\ j \neq i}}^{M} A_{i j} x_{j}(t), i=1, \ldots, M \text { for all } t
$$

Similar to [5], the constraints (22) are relaxed by introducing the corresponding Lagrange multipliers $p_{i} \in \mathfrak{R}^{n_{i}}$ (also referred to as prices) in the cost function (14). Further, define the following sequences:

$$
\begin{gathered}
P=\left[p_{t}, p_{t+1}, \ldots, p_{t+N}\right] \\
p_{t+k}=\left[p_{1, t+k}, p_{2, t+k}, \ldots, p_{M, t+k}\right], k=0,1, \ldots, N \\
V=\left[v_{t}, v_{t+1}, \ldots, v_{t+N}\right], V_{i}=\left[v_{i, t}, v_{i, t+1}, \ldots, v_{i, t+N}\right] \\
v_{t+k}=\left[v_{1, t+k}, v_{2, t+k}, \ldots, v_{M, t+k}\right], k=0,1, \ldots, N
\end{gathered}
$$

Then, the original problem $\mathrm{P} 1$ is reformulated as a dual problem in view of distributed robust MPC:

\section{Problem P2 (distributed min-max MPC)}

$$
\begin{aligned}
& x_{i, t+k+1 \mid t}=A_{i} x_{i, t+k \mid t}+B_{i} u_{i, t+k}+v_{i, t+k},\left[A_{i}, B_{i}\right] \in \tilde{\Omega}_{i} \\
& k=0,1, \ldots, N-1
\end{aligned}
$$

Let $G_{i}^{*}=\left[G_{i, t}^{*}, G_{i, t+1}^{*}, \ldots, G_{i, t+N}^{*}\right], g_{i}^{*}=\left[g_{i, t}^{*} g_{i, t+1}^{*}, \ldots, g_{i, t+N}^{*}\right]$ and $V_{i}^{*}=\left[v_{i, t}^{*} v_{i, t+1}^{*}, \ldots, v_{i, t+N}^{*}\right]$ be the optimal solution of the

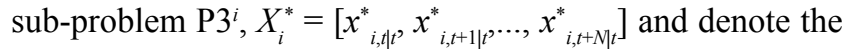
worst-case state trajectorycorresponding to the optimal solution $G_{i}^{*}, g_{i}^{*}, V_{i}^{*}$, i.e.

$$
\begin{aligned}
& x_{i, t+k+1 \mid t}^{*}=A_{i}^{*} x_{i, t+k \mid t}^{*}+B_{i}^{*}\left(G_{i, t+k} x_{i, t+k \mid t}^{*}+g_{i, t+k}\right)+v_{i, t+k}^{*} \\
& k=0,1, \ldots, N-1
\end{aligned}
$$

where

$$
\begin{aligned}
& {\left[A_{i}^{*}, B_{i}^{*}\right]=} \\
& \arg \max _{\left[A_{i}, B_{i}\right] \in \tilde{\Omega}_{i}} \sum_{k=0}^{N} l_{i}^{P}\left(G_{i, t+k}^{*}, g_{i, t+k}^{*}, v_{i, t+k}^{*}, P, A_{i}, B_{i}\right)
\end{aligned}
$$

It can be easily shown that under Assumption A3, the optimization problem P1 (where the equation (7) is represented equivalently as equation (20)) is convex with respect to the optimization variables $G$ and $g$. Then it can be proved that if $x=\left[x_{1}, x_{2}, \ldots, x_{M}\right]$ is a feasible initial state of the system (1) (i.e. state for which there exists a sequence $\mathrm{F}=\left[F_{1}, F_{2}, \ldots, F_{M}\right]$ of feedback laws such that all constraints in the problem $\mathrm{P} 1$ are satisfied), then it holds:

$$
\text { (36) } \quad V^{*}(x)=\max _{P} \sum_{i=1}^{M} V_{i}^{*}\left(P, x_{i}\right) \text {, with } p_{t+N}=0
$$

It means that the solution of the centralized min-max MPC problem P1 is equivalent to the solution if the distributed dual min-max MPC problem P2. The proof of (36) follows similar arguments as in [12].

From (36) it follows that the computation of $G_{i}^{*}, g_{i}^{*}, V_{i}^{*}$ for given prices $P$ can be done in a decentralized way, but finding the optimal prices requires coordination. The prices are found by applying the accelerated proximal gradient method to solve the dual problem to a convex primal optimization problem (see [16] and the references therein). Given a price prediction sequence $P^{r}=\left[p_{t}^{r}, \ldots, p_{t_{+N}}^{r}\right]$ for the $r$-th iteration, the corresponding sequences

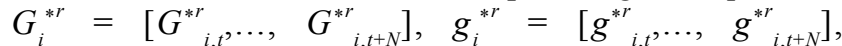

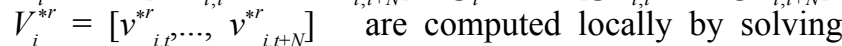
problem $\mathrm{P} 3^{i}$ and (34)-(35). Then, the prices can be updated distributedly with the following iteration for the $i$-th subsystem:

$$
\begin{aligned}
& q_{i, t+k}^{r}=p_{i, t+k}^{r}+\frac{r-1}{r+2}\left(p_{i, t+k}^{r}-p_{i, t+k}^{r-1}\right), k=0,1, \ldots, N-1 \\
& p_{i, t+k}^{r+1}=q_{i, t+k}^{r}+\left.\frac{1}{L} \nabla_{P_{i}} S(P, x)\right|_{P=Q^{r}}, k=0,1, \ldots, N-1 \\
& \text { with } p_{i, t+N}^{r+1}=p_{i, t+N}^{r}=0
\end{aligned}
$$

where $Q^{r}=\left[Q_{1}^{r}, Q_{2}^{r}, \ldots, Q_{M}^{r}\right], Q_{i}^{r}=\left[\begin{array}{lll}q^{r}{ }_{i, t}, \ldots, & q^{r}{ }_{i, t+N}\end{array}\right]$, $i=1,2, \ldots, M$ (with $q_{i, t+N}^{r}=p_{i, t+N}^{r}=0$ ), and $S(P, x)$ is the dual function (cf. (24)): 


\section{$S(P, x)=\min _{G, g, V} \max _{\substack{\left[A_{i}, B_{j}\right] \in \tilde{S}_{i} \\ i=1,2, \ldots, M}}$ \\ (38) $\sum_{k=0}^{N} \sum_{i=1}^{M}\left[l_{i}\left(x_{i, t+k \mid t}, G_{i, t+k}, g_{i, t+k}\right)+p_{i, t+k}^{T}\left(v_{i, t+k}-\sum_{\substack{j=1 \\ j \neq i}}^{M} A_{i j} x_{j, t+k \mid t}\right)\right]$}

The gradient of the dual function $S(P, x)$ with respect to the prices $P_{i}$ at $P=Q^{r}$ is

$$
\begin{aligned}
& \left.\nabla_{P_{i}} S(P, x)\right|_{P=Q^{r}}=v_{i, t+k}^{*}-\sum_{\substack{j=1 \\
j \neq i}}^{M} A_{i j} x_{j, t+k \mid t}^{*} \\
& k=0,1, \ldots, N-1
\end{aligned}
$$

From (39), it can be seen that in order to compute the gradient $\left.\nabla_{P_{i}} S(P, x)\right|_{P=Q^{r}}$ in (37) it is necessary to have the worst-case state trajectories $X_{j}^{*}=\left[x_{j, t \mid t}^{*}, x_{j, t+1 \mid t}^{*}, \ldots, x_{j, t+N \mid t}^{*}\right]$, $j=1, \ldots, M, j \neq i$ of the interconnected subsystems, which on their hand depend on the values $P_{i}$ of prices.

In (37), $L$ is the Lipschitz constant to the gradient function $\nabla_{P} S(P, x)$. In [12], an off-line algorithm to obtain an estimate of $L$ is provided.

The following suboptimal algorithm to distributed closed-loop min-max MPC of uncertain polytopic systems is proposed.

\section{Algorithm 1 (Distributed closed-loop min-max MPC by on-line optimization)}

1. Obtain an estimate $\hat{L}$ of the Lipschitz constant to the gradient $\nabla_{P} S(P, x)$, number $R$ of iterations, and fix arbitrary guesses $P_{i}^{0}, i=1,2, \ldots, M$ for the price sequences. Let $t=0$.

2. Let the state at time $t$ be $x(t)=x=\left[x_{1}, \ldots, x_{M}\right]$.

3. for $r=0,1, \ldots, R-1$ do

4. For $i$-th subsystem, $i=1,2, \ldots, M$, communicate the price sequences $P_{j}^{r}=\left[p_{j, t}^{r}, \ldots, p_{j, t+N}^{r}\right], j=1, \ldots, M, j \neq i$ of the interconnected subsystems.

5. Compute the sequences $G_{i}^{* r}=\left[G_{i, t}^{* r}, \ldots, G_{i, t+N}^{* r}\right]$, $g_{i}^{*_{r} r}=\left[g_{i, t}^{*_{r}}, \ldots, g_{i, t+N}^{*_{r}}\right]$ and $V_{i}^{*_{r}}=\left[v_{i, t}^{*_{r}}, \ldots, v_{i, t+N}^{*_{r}}\right]$, corresponding to the price sequence $P^{r}=\left[p_{t}^{r}, \ldots, p_{t+N}^{r}\right]$ by solving distributedly the QP sub-problems $\mathrm{P}^{i}, i=1,2, \ldots, M$. Compute the worst-case state trajectories $X_{i}^{* r}=\left[x_{i, t \mid t}^{*_{r}}, \ldots, x_{i, t+N \mid t}^{* r}\right], i=1,2, \ldots, M$ from (34)-(35).

6. For $i$-th subsystem, $i=1,2, \ldots, M$, communicate the worstcase state trajectories $X_{j}^{*_{r}}=\left[x_{j, t \mid t}^{*_{r}}, \ldots, x_{j, t+N \mid t}^{*_{r}}\right], j=1, \ldots, M$, $j \neq i$ of the interconnected subsystems.

7. Compute distributedly the updates $P_{i}^{r+1}=\left[p_{i, t}^{r+1}, \ldots, p_{i, t+N}^{r+1}\right], i=1,2, \ldots, M$ of the price sequences by applying (37) with $L=\hat{L}$ and using (39).
8. end

9. Let $P_{i}^{0}=P_{i}^{R}, i=1,2, \ldots, M$.

10. Apply to the overall system the control inputs $u_{i}(t)=u_{i, t}^{* R-1}, i=1,2, \ldots, M$.

11. Let $t=t+1$ and go to step 2 .

It is clear that the computational load required by the centralized solution of the robust MPC problem will increase rapidly both with the increase of the number of the subsystems constituting the overall system and with their dimensions. In contrast, with the distributed robust MPC approach the original, large size, optimization problem is decomposed into a number of smaller and more tractable ones, which can be solved in parallel. Therefore, the computational complexity depends only on the number of the variables and the number of the constraints associated to each subsystem. Another advantage of the distributed approach is that it allows the performance optimization of the subsystems to be done autonomously and it is not required to have a central optimization unit. In addition, the distributed MPC is characterized with reduced communicational requirements, because only the interconnected subsystems need to communicate in order to optimize the global performance.

With the proposed robust MPC approach the purpose is to achieve robust feasibility (satisfaction of the constraints for all possible uncertainties within the uncertainty set) and robust performance (optimizing the worstcase value of the performance index). It is known that the robust control system may have optimal performance only for some values of the uncertain parameters, i.e. the optimality is "sacrificed" in some extent in order to achieve robustness. It has to be kept in mind that if a nominal MPC controller is designed by ignoring the presence of uncertainty, this will lead to violation of constraints and possible poor performance for values of the uncertain parameters, which differ from the nominal ones. Therefore, it is necessary to guarantee robustness of the control system when there is uncertainty about plant dynamics. By using the closed-loop min-max MPC approach to obtain robustness, a less conservative solution is obtained in comparison to the open-loop min-max approach. The price to be paid for this less conservative solution is an increased computational complexity for the closed-loop predictions.

\section{Simulation Example}

Consider the following system composed of two interconnected polytopic subsystems $\mathrm{S}_{1}$ and $\mathrm{S}_{2}$ : Consider the following system composed of two interconnected polytopic subsystems and $S_{2}$ :

$$
\begin{aligned}
S_{1}: & x_{1}(t+1)=A_{1}(t) x_{1}(t)+B_{1} u_{1}(t)+A_{12} x_{2}(t) \\
& A_{1}(t) \in \Omega_{1}
\end{aligned}
$$




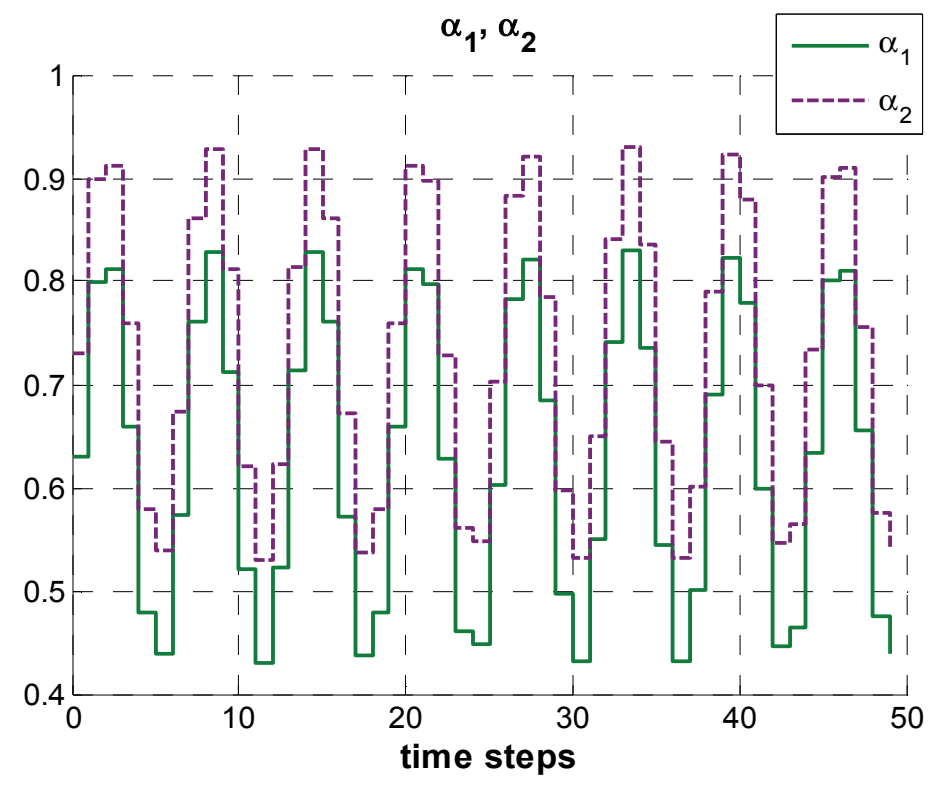

Figure 3. The variation of parameters

$$
\text { (41) } \begin{gathered}
S_{2}: \\
x_{2}(t+1)=A_{2}(t) x_{2}(t)+B_{2} u_{2}(t)+A_{21} x_{1}(t) \\
A_{2}(t) \in \Omega_{2}
\end{gathered}
$$

where

(42)

$$
\begin{aligned}
& A_{1}(t)=\left[\begin{array}{cc}
\alpha_{1}(t) & -0.09 \\
0.17 & 0.79
\end{array}\right], \alpha_{1}(t) \in[0.43,0.83] \\
& A_{2}(t)=\left[\begin{array}{cc}
\alpha_{2}(t) & -0.09 \\
0.17 & 0.69
\end{array}\right], \alpha_{2}(t) \in[0.53,0.93] \\
& B_{1}=\left[\begin{array}{l}
0.06 \\
0.01
\end{array}\right], B_{2}=\left[\begin{array}{l}
0.07 \\
0.01
\end{array}\right] ; A_{12}=A_{21}=\left[\begin{array}{cc}
0 & 0 \\
0 & 0.1
\end{array}\right]
\end{aligned}
$$

Here, $\alpha_{1}$ and $\alpha_{2}$ are uncertain parameters. The sets $\Omega_{1}$ and $\Omega_{2}$ have two vertices corresponding to $\alpha_{1}=0.43$, $\alpha_{1}=0.83$ and $\alpha_{2}=0.53, \alpha_{2}=0.93$, respectively. The finite sets $\tilde{\Omega}_{1}$ and $\tilde{\Omega}_{2}$ are defined as:

$$
\widetilde{\Omega}_{1}=\left\{\left[A_{1}\left(\alpha_{1}\right), B_{1}\right], \alpha_{1} \in\{0.43,0.53,0.63,0.73,0.83\}\right\}
$$

$$
\widetilde{\Omega}_{2}=\left\{\left[A_{2}\left(\alpha_{2}\right), B_{2}\right], \alpha_{2} \in\{0.53,0.63,0.73,0.83,0.93\}\right\}
$$

The following state and input constraints are imposed on the system (40)-(43):

$$
\text { (44) }\left[\begin{array}{l}
-0.1 \\
-0.1
\end{array}\right] \leq x_{i}(t),-2 \leq u_{i}(t) \leq 2, i=1,2
$$

The prediction horizon is $N=5$ and the weighting matrices are $Q_{i}=I, R_{i}=0.01, i=1,2$. The centralized closedloop min-max MPC problem (problem P1) is represented as a distributed problem (problem P2) by applying the dual decomposition approach. Then, Algorithm 1 with number of iterations $R=10$ is used to generate the two control inputs for an initial state of the overall system $x(0)=\left[\begin{array}{lll}2 & 2 & 2\end{array}\right]$. The simulations are performed for the variations of the uncertain parameters, shown in figure 3.
The computed trajectories of the control inputs $u_{1}, u_{2}$ and the states $x_{1}{ }^{1}, x_{2}{ }^{1}$ and $x_{1}{ }^{2}, x_{2}{ }^{2}$, associated to the subsystems $S_{1}$ (i.e. $x_{1}=\left[x_{1}^{1}, x_{2}^{1}\right]$ ) and $S_{2}$ (i.e. $x_{2}=\left[x_{1}^{2}, x_{2}^{2}\right]$ ) are depicted in figure 4 to figure 6 . The results show that the suboptimal trajectories obtained with the distributed min-max MPC keep both the state and input constraints.
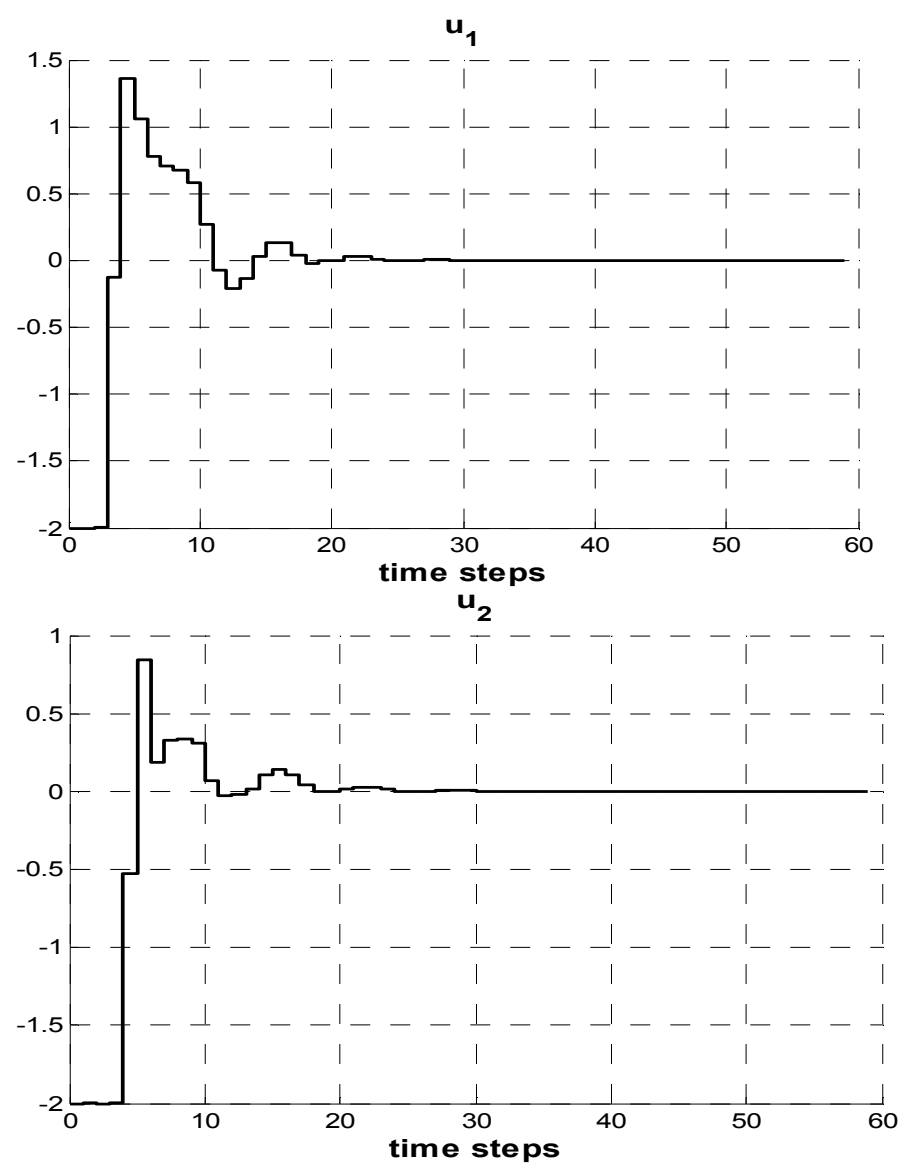

Figure 4. The control inputs $u_{1}, u_{2}$ 


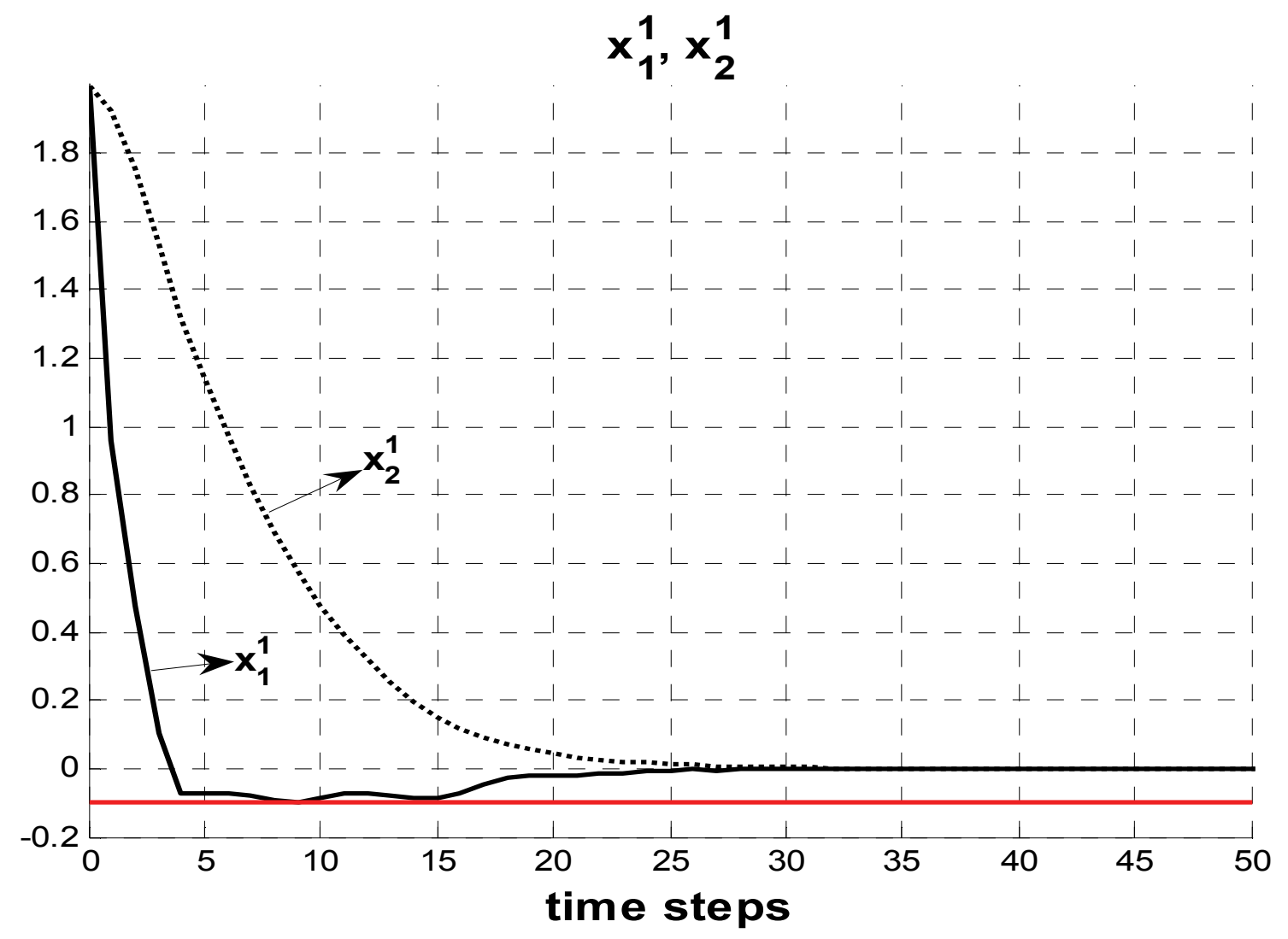

Figure 5. The states $x_{2}^{1}, x_{2}^{2}$ of subsystem $S_{1}$

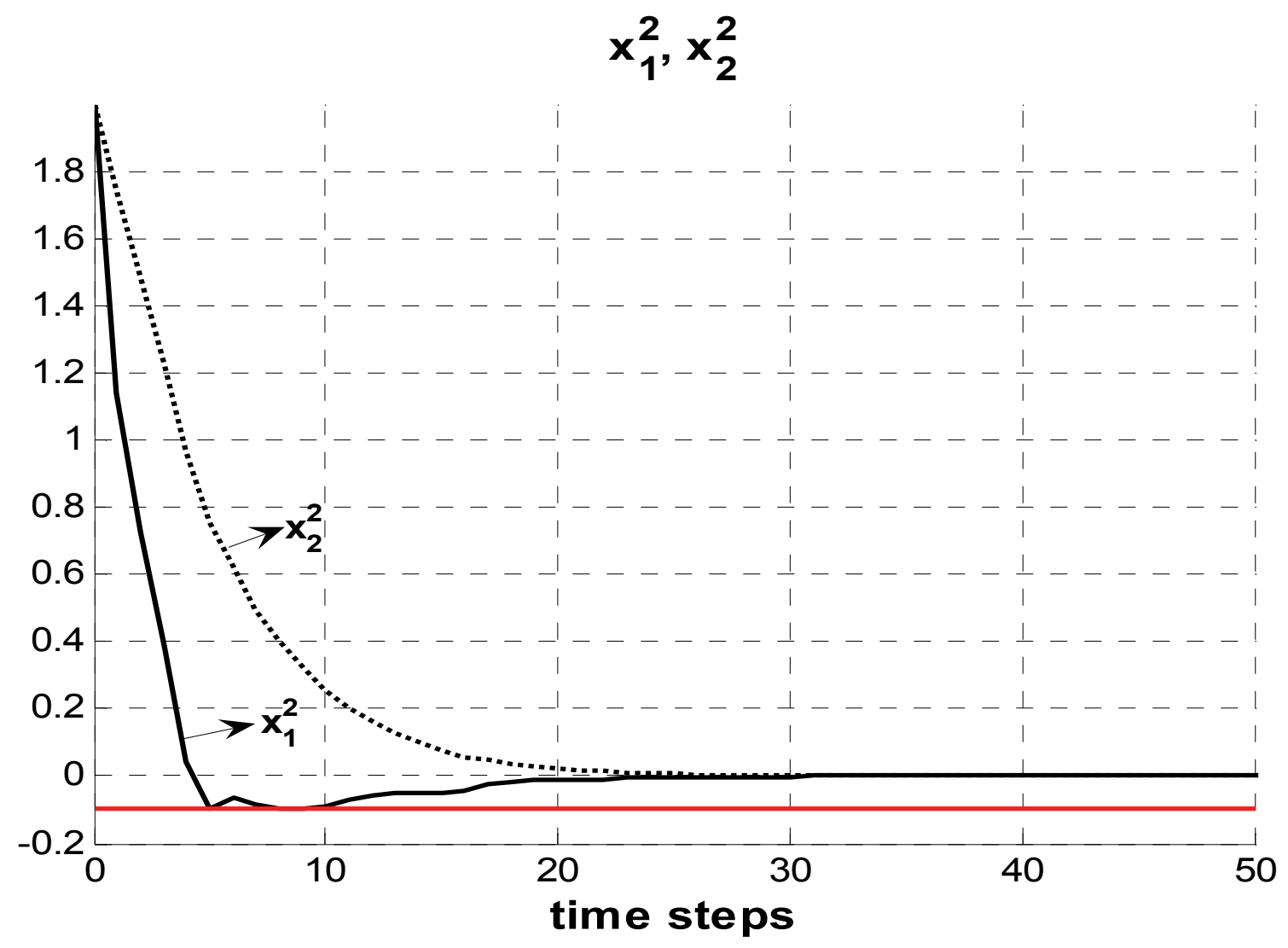

Figure 6. The states $x_{2}^{1}, x_{2}^{2}$ of subsystem $S_{2}$ 


\section{Conclusions}

In this paper, a suboptimal approach to distributed closed-loop min-max MPC for uncertain systems consisting of polytopic constrained subsystems is proposed and its performance is illustrated with a numerical example. The results show that the suboptimal trajectories obtained with the proposed approach keep both the state and input constraints.

\section{References}

1. Bemporad, A., M. Morari. Robust Model Predictive Control: A Survey. Garulli, A., A. Tesi, A. Vicino (Editors). Robustness in Identification and Control, number 245 in Lecture Notes in Control and Information Sciences, Springer-Verlag, 1999, 207-226.

2. Mayne, D. Q., J. B. Rawlings, C. V. Rao, P. O. M. Scokaert. Constrained Model Predictive Control: Stability and Optimality. Automatica, 36, 2000, 789-814.

3. Maciejowski, J. M. Predictive Control with Constraints. Pearson Education Limited, 2002.

4. Camacho, E. F., C. Bordons. Model Predictive Control. 2nd Ed., Springer-Verlag, 2004

5. Campo, P. J., M. Morari. Robust Model Predictive Control. Proceedings of the American Control Conference, 1987.

6. Magni, L., R. Scattolini. Robustness and Robust Design of MPC for Nonlinear Discrete-time Systems. Findeisen, R., F. Allgoewer, L. Biegler (Eds.). Assessment and Future Directions of Nonlinear Model Predictive Control. - LNCIS, 358, 2007, 239-254, Springer, Heidelberg,

7. Scattolini, R. Architectures for Distributed and Hierarchical Model Predictive Control - A Review. - Journal of Process Control, 19, 2009, 723-731.

8. Christofides, P. D., R. Scattolini, D. Muñoz de la Peña, J. Liu. Distributed Model Predictive Control: A Tutorial Review and Future Research Directions. - Computers and Chemical Engineering, 51, 2013, 21-41.

9. Maestre, J. M., R. R. Negenborn. Distributed Model Predictive Control Made Easy. Series: Intelligent Systems, Control and Automation: Science and Engineering, 69, Springer-Verlag, 2014. 10. Zhang, L., J. Wang, C. Li. Distributed Model Predictive Control for Polytopic Uncertain Systems Subject to Actuator Saturation. Journal of Process Control, 23, 2013, 1075-1089.

11. Al-Gherwi, W., H. Budman, A. Elkamel. A Robust Distributed Model Predictive Control Algorithm. - Journal of Process Control, 21, 2011, 1127-1137.

12. Grancharova, A., S. Olaru. An Approach to Distributed Robust Model Predictive Control of Discrete-time Polytopic Systems. Proceedings of the 19-th IFAC World Congress, Cape Town, South Africa, 2014, 2576-2581.

13. Grancharova, A., S. Olaru. Distributed Robust Model Predictive Control of Interconnected Polytopic Systems. S. Olaru, A. Grancharova, F. Pereira (Editors). Developments in Model-Based Optimization and Control: Distributed Control and Industrial Applications, Springer-Verlag, 2015, 73-91.

14. Cohen, G., B. Miara. Optimization with an Auxiliary Constraint and Decomposition. - SIAM Journal on Control and Optimization, 28, 1990, 137-157.

15. Giselsson, P., A. Rantzer. Distributed Model Predictive Control with Suboptimality and Stability Guarantees. Proceedings of the 49-th IEEE Conference on Decision and Control, Atlanta, GA, 2010, 7272-7277.

16. Giselsson, P., M. D. Doan, T. Keviczky, B. De Schutter, A. Rantzer. Accelerated Gradient Methods and Dual Decomposition in Distributed Model Predictive Control. - Automatica, 49, 2013, 829-833.

\section{Manuscript received on 27.01.2016}

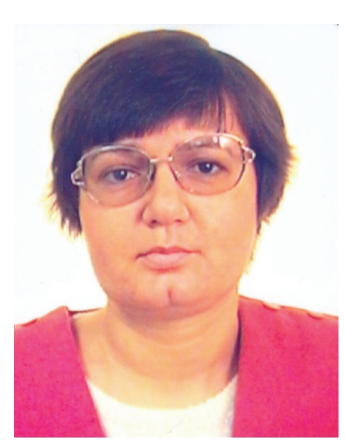

Alexandra Grancharova is an Associate Professor at the Department of Industrial Automation, University of Chemical Technology and Metallurgy (UCTM), Sofia, Bulgaria. She received her M.Sc. and Ph.D. degrees in Automation of Industrial Processes from UCTM. She co-authored one Springer monograph (Explicit Nonlinear Model Predictive Control: Theory and Applications, LNCIS, vol. 429) and co-edited one Springer book volume (Developments in Model-Based Optimization and Control: Distributed Control and Industrial Applications, LNCIS, vol. 464). She published two textbooks for the students at UCTM (Model-based Control and Optimal and Robust Systems, in Bulgarian). Beside that, Grancharova has more than 110 publications in the fields of optimal control, explicit model predictive control, distributed model predictive control, and modeling, simulation and optimal control of industrial processes. She has been the coordinator of two international projects and has participated in several EU funded research projects.

Contacts:

Department of Industrial Automation University of Chemical Technology and Metallurgy $8 \mathrm{Kl}$. Ohridski Blvd., 1756 Sofia, Bulgaria e-mail: alexandra.grancharova@abv.bg

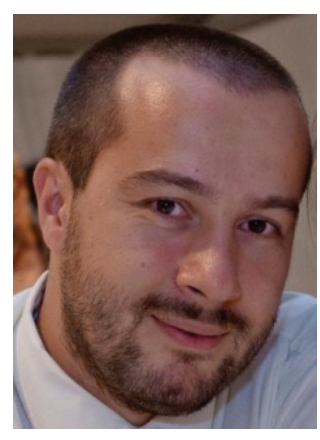

Sorin Olaru is a Professor in the Automatic Control Department of CentraleSupélec, member of the CNRS Laboratory of Signals and Systems and associate researcher in the INRIA team DISCO, all institutions being part of the university Paris-Saclay in France. He received the PhD from Paris XI University in 2005, the PhD from University "Politehnica" in Bucharest in 2010 and the French "Habilitation à diriger des recherches" in 2011, being also the recipient of the European Commission's Archimedes Prize in 2002. His research is related to constrained control, network control systems, fault tolerant control, uncertainty characterization in $d y$ namical systems and control of systems affected by delays. He held visiting appointments at KIT Fukuoka (Japan), NTNU Trondheim (Norway), CSDC Newcastle (Australia), Bernoulli Center in Lausanne (Swiss) and Linnaeus Center in Lund (Sweden). He is a Senior member of IEEE, member of IFAC Technical Committee on Robust Control, Associate Editor for IMA Journal of Mathematical Control and Information, coordinator of six international cooperative research projects and participant in three European research projects (one as coordinator and two as national representative and principal investigator). He co-authored a monograph (Set-Theoretic Fault Tolerant Control in Multisensor Systems. ISTE - Hermes, John Wiley \& Sons, 2013), one book in the SpringerBriefs series (MixedInteger Representations in Control Design, Springer 2015), 20 book chapters, 35 journal publications and more than 120 conference papers.

Contacts:

Laboratory of Signals and Systems CentraleSupélec-CNRS-Université Paris-Sud Université Paris-Saclay

3 Rue Joliot-Curie, 91192 Cedex Gif-sur-Yvette, France e-mail: Sorin.Olaru@centralesupelec.fr 